\title{
Evaluate Legal, Operational and Governance Framework Design from Whitepapers for Initial Coins Offer (ICO)
}

\author{
Wong Wai Wai, Muhammed Siraj
}

\begin{abstract}
In this 20th Century Digital World, the Initial Coins Offer (ICO) acts as fundraisers for a start-up that is related to technology. There have been many successful ICOs launches over the past several years that have resulted in giving investors good returns for their investments. However, the lack of regulatory framework has resulted in the unregulated position and the unidentified nature of the dealings involved. There is a substantial number of ICOs which were scams and have resulted in multi-million-dollar fraud schemes. Potential Investors of ICOs are presently susceptible to misrepresentations, scam, and exploitation. Unlike the traditional mode of fundraising such as Initial Public Offer (IPO) where investors can access information through the vetted official prospectus of the proposed company which includes past financial performance and projected future operations together with all related financial statements. However, ICO potential investors for proposed ICO projects are only able to access and scrutinize published ICO whitepapers. ICO whitepapers are the only available documents or the source information that investors can access and rely on to make an informed judgment and investment decision. This research paper will highlight the important areas that should be rigorously studied and verified by potential investors when making an investment decision and to identify and distinguish the indicators of scam or fraudulent ICO project. Potential investors should familiarise themselves with the various types of ICO token and property rights attached accordingly as each token possesses a unique bundle of property rights. The property rights of ICO token together with the ERC-20 token standard which consists of six mandatory rules would serve as a useful guide for the potential investor to understand and to evaluate the legal, operational and governance of the framework of ICO projects in order to make an informed and appropriate investment decision.
\end{abstract}

Index Term: Initial Coin Offer, Property Rights, ERC-20 Token Standards.

\section{INTRODUCTION}

Initial Coins Offers (ICO) are equivalent to an Initial Public Offers (IPO) in the mainstream regulated investment world. ICO is a platform for fundraising for starts up businesses that are related to technology. There are many examples of successful ICOs. The most profitable ICOs in past several years have rewarded investors with good returns for their investments. However, investors' enthusiasm has also led people off course. Because ICOs are mostly non-regulated, the ICOs have developed into a hub for fraudsters and con artists, seeking for opportunities to target

Revised Manuscript Received on September 22, 2019.

Wong Wai Wai, University of Malaya, Faculty of La. drwongwaiwai@gmail.com

Muhammed Siraj, Universiti Putra Malaysia on unsuspecting investors who are under-informed and eager to make quick and substantial returns.

Potential investors of ICOs are presently susceptible to misrepresentations, scam, and manipulations. Many ICOs are not subjected to the respective laws and authorities because of the way they structured their project and the nature of the token they offer. ICOs provides an ideal platform for the laundering of criminal activities. Potential investors of ICOs are blind to these risks. (1)(2). When the potential investors are not aware of these risks and take no precautionary measure, there is a high probability that investors will lose their whole asset. For example, the Securities \& Exchange Commission of the United States has brought criminal charges against two ICOs issuers (REcoin Group Foundation and DRC World) on the basis that they have supplied bogus and deceiving data to investors. (3). They have made a false promise on high returns from activities relating to real estate and diamonds. According to the SEC's protest, investors were told to expect high returns from the company business. The untrue statements made by REcoin such as "The First Ever Real Estate Backed Cryptocurrency" as well as "group of attorneys, professionals, stock brokers, and accountants" but in fact, none of the specialists had been hired or even referred at all. Zaslavskiy and REcoin misrepresentation allegedly has raised between $\$ 2$ million and $\$ 4$ million from investors when the actual amount was approximately $\$ 300,000 . ”(3)$

Many investors allowed themselves to be attracted by the assurance of huge returns, but they are not fully mindful that the presented ICO project is a new development that is still very much in its infancy stage. The reality is that the ICO project is something that is very new. It is highly uncertain whether the provider of a proposed ICO project can actually carry out the promised plans. Even if the plan is executed and carry through, there is no guarantee that the eventual worth of the product or service in the ICO project will be equivalent to the amount that has been invested by the investors.(4) Unlike the traditional established format, like the IPO where an investor is able to view information from the circulated vetted prospectus regarding the company which would necessarily include past financial performances and expected future operations together with the appropriate financial statements. It is very important that potential investors of ICOs do read and understand ICO whitepapers carefully. It is because ICO whitepapers are the only available main and primary document or information that investors can rely on in making an informed judgment and better investment decision.(4)

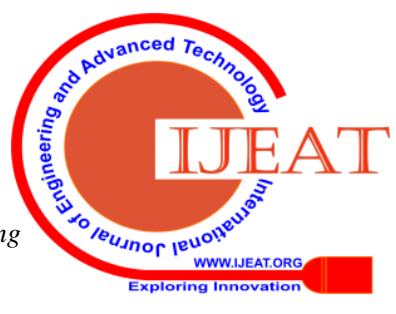


The objective of this research paper is to identify the important areas that ought to be rigorously studied and verified by potential investors before committing themselves to make an investment decision in ICO. It would include looking at the various issues such as the team composition, the problem, the proposed solution, token value, Community and media and so on. Some information requires serious attention such as the detailed technical description of the project, token implementation, token distribution, sale mechanism, and issues in relation to legality. Most potential investors often miscalculate the technical knowledge and capability required to be able to read and understand the whitepapers of ICO in order to make a knowledgeable and learned decision for this type of financing. Therefore, this research paper will focus on two important areas concerning the legal, operational and governance framework design of ICOs. These areas although it requires some technical knowledge will be able to give an insight into the infrastructure and governance framework of ICO project. The discussions in this research paper will be divided in the following manner:

- Blockchain, Cryptocurrency, and Token

- Introduction to ICO and its Structure

- Risks Associated with ICOs

- Key Points to Observe in ICOs Whitepapers

o The types of ICO tokens and its legal status of ICO token

o Analysis of various property rights of actual ICO tokens

oOperational and Governance Framework Design

○Conclusion

\section{BLOCKCHAIN, CRYPTOCURRENCY AND TOKEN}

Before we examine the issues concerning ICOs and its whitepapers, one must be able to understand the various terms that are often used in this field. Blockchain technology is a transformative new technology that can revolutionize the way we conduct business. The idea of blockchain was presented some ten years ago by Satoshi Nakamoto, the architect of the first cryptocurrency, Bitcoin.(5). Bitcoin is an implementation of electronic cash that need not be authorized or supervised by or conducted through the relevant country's Monetary Central Authority or licensed Banks. However, there is a lot of confusion as to what exactly is meant by blockchain and cryptocurrency. The confusion seems to originate from the usage of the terms. Blockchain is the underlining technology engaged. Blockchain is a digitized, distributed, public ledger multiplying on blocks which are logged and added to it in sequential order by actors or participants known as miners or block producers. Decentralized Ledger Technology (DLT) is the common term used to describe the methods of preserving dispersed ledgers in the networks of computers.(6) (Majaski, 2018) The term 'blockchain' often used as a substitute for DLT is not accurate by reason that blockchain embodies only one type of DLT.(8) Several of the best-known occurrences of DLT are based on blockchain approach, but there are other categories that are not constructed on blockchain technology. Examples of these other DLT technologies are Hashgraph, IOTA amongst others. The variance between blockchain and DLT is that DLT is a broad phrase used to explain technologies that disseminate records or data between those who are using it, whether privately or openly. Blockchain is just a sub-classification of this broad term DLT. (8).

Whereas, Cryptocurrency is a digital asset that has to do with the use of tokens based on DLT. Cryptocurrency is used as a instrument or means on a blockchain web for the purpose of buying, selling, investing or trading with goods or services.(9) Cryptocurrency is a digital currency that is based on a technology called cryptography. The usage of cryptography is for security. (10) Cryptography is simply defined as the usage of maths to encode and decode data. (10) Cryptography enables users to store confidential data or transfer it through the Internet. No one will be able to read the data except by the intended recipient. Cryptocurrency by using cryptography is to protect the intended data and communications via the use of codes. The code will render only those for whom the data is targeted to be able to process and to read it. (11) It is a communication technique that is developed from mathematical models and a set of rule-based computations. Algorithms are utilized to convert messages in ways that are challenging to decode.(11) These deterministic algorithms are utilized for cryptographic key creation. Furthermore, it is also used for digital signing and authentication to safeguard data confidentiality between the various parties in a transaction. It has been applied in web surfing on the internet and classified communications in credit card operations and email. (11) A cryptocurrency is challenging to counterfeit for of its security attributes. (12) Bitcoin is the first cryptocurrency created and it is only one of the cryptocurrencies that are available in the market.

There is a distinction between cryptocurrencies and tokens. Cryptocurrencies are digital exchanges that are coded (secured) applying cryptography which is used to secure and verify transactions. (13) For example, Bitcoin is the first distributed cryptocurrency, which is operated by a public ledger that documents and substantiates all dealings sequentially, called the Blockchain. (13) A token is a form of cryptocurrency. Tokens require a platform such as Ethereum or Omni to exist and operate.(13) The token is used to fund the operating expenses required to host it as a service, such as a ICO project, whereas cryptocurrencies can be described as a medium of exchange.(14)

\section{INTRODUCTION TO ICO AND ITS STRUCTURE}

ICO is a type of cryptocurrency that has been specifically created for a purpose that is very similar to an IPO in the mainstream investment world. An ICO is a new way to raise capital for business.(15) The sale of ICO token is a sale of unregulated digital asset that represents the potential value of an early-stage project or concept.(16) This token sale is a new method that differs significantly from existing fundraising mechanisms. It is more akin to venture capital than the traditional fundraising method that allows a private company to go public. (16) Some of the noticeable differences

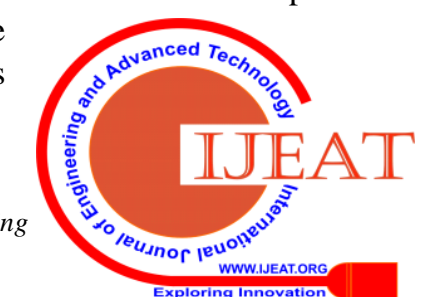


between ICO and IPO are as follows: First, the company or startup seeking to raise capital does not need to issue shares, bonds or any other form of financial products. Secondly, the issuer of ICO does not receive money in the traditional sense. The issuer receives crypto-assets that are accepted by the public such as Bitcoin or Ether. Thirdly, the issuance of ICO is not issued by the central authority or investment bank. There is no intermediary body involved. Fourthly, the issuance of tokens does not require the preparation and registration of a prospectus, unless tokens are considered as a form of securities under traditionally established securities law. Therefore, all that is required is to prepare a simple document called a "white paper" which primarily discloses all the relevant information and obligations to the potential investors of ICO. It is important to take note that there is no existing comprehensive law in any country that currently governs or regulates the contents of ICOs whitepapers. Due to the unregulated position and the unidentified description of the dealings engaged in this new fundraising method and technology, ICOs are becoming increasingly attractive for those wanting to engage in illegal methods. The present propaganda surrounding cryptocurrency and ICOs may blind investors to these consequences. (2) The type of risks involved in investing ICOs will be further elaborated in the later part of this research paper.

Structure of ICO should consist of the following headings:

- Investor

- Team

- Product

- Audience

- Market

- Risk

- Warranties

- Metrics

- Budget

- Reporting

- Compliance Governance

\section{RISKS ASSOCIATED WITH ICOS}

As ICOs are not subject to any substantive laws and regulations, they are an attractive model for money laundering and fraud. As ICOs are mostly unregulated, they expose unsuspecting investors to risks and investors may lose their entire investment. For example, the Securities \& Exchange Commission has charged the issuers of two ICOs, namely the REcoin Group Foundation and DRC World for providing false and misleading information to investors. In relation to their company's activities. (3)

Another ICO project that has been busted by law enforcement in China for soliciting money from investors with fraudulent claims that involve $\$ 47$ million. (17) The project's website states that buyers of the token are entitled to hold a contract representing ownership of a certain amount of the Pu'er Tibetan teas in stock which it claims to be worth billions of dollars and generate high short-term returns to investors. However, all these claims turned out to be untrue.(17)

Many investors allow themselves to be persuaded by the guarantee of ridiculous high returns of the ICO projects.
What potential investors do not realize is that these projects are still very much in its infancy stage. The reality is that the ICO project is something that is very new. It is highly uncertain whether the provider of an ICO project will actually carry out the plan. Even if the plan has been carried out and executed, there is no promise that they can actually earn according to what has been projected.

\section{A. 'White Paper' is Described as:}

"an influential statement or guideline that notifies readers succinctly about a complicated problem and introduces the issuing entity's viewpoint on the subject. It is intended to assist readers comprehend a matter, work out a question, or make a judgment." (18)

In many cases, the sources of ICOs are frequently not evident in providing information to potential investors. Important and rudimentary information such as the background of the company, who is really behind the project, the risks involved in the venture, the privileges of the proprietor of the tokens or how the funding will be handled often explained in very brief words. According to this study, $19 \%$ of the sample white papers offer simply technical knowledge about the product or procedure that is to be established. (19) $21 \%$ of the white papers do not offer any info about the originators or sponsors of the ICO projects, $25 \%$ of the white papers do not provide any explanation or details of the project's fiscal circumstances and how the capital collected is to be used or distributed, $82 \%$ of white paper is quiet on whether the financing to be given by numerous applicants will be merged or stay separated. (19) In most ICOs that were within the sampling in this study, potential participants are given insufficient financial information. It is difficult for potential investors to make an investment decision based on a rational calculus.(19) There is also no external audit to certify the information presented in whitepapers. Without all these essential and basic information, it is virtually impractical for potential investors to evaluate the true worth of ICOs and to differentiate authentic ICOs from illegal schemes. This absence of clarity is a major hurdle to the efficiency of the pricing of ICO tokens.

\section{KEY POINTS TO OBSERVE IN ICOS WHITEPAPERS}

Therefore, it is important that all potential investors should read, understand, study and scrutinize the whitepapers carefully before making the decision to participate in an ICO project. Important areas to note such as product, team, target users, problems vs solution, token value, token distribution, road map, use of funds, terms and conditions that should all be clearly explained in whitepapers. On top of that, there are two further main crucial areas that the potential investors should focus on: (i) the legal status of ICO token and its property rights; and (ii) Operational and Governance Framework Design.

\section{A. The Types of ICO Tokens, its Legal Status of ICO} Token

Before examining the legal status and property rights of

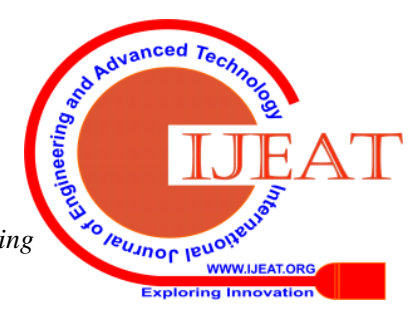


ICO token, one need to appreciate there are different classes of ICO tokens and each of them possesses its own unique characteristic.

\section{1) Utility Tokens}

As the name suggests, utility tokens offer a customer to utilize a blockchain-based program that provides a certain service. Utility tokens offer the owner with the mandate to be present within a program.(20) Utility tokens are like App coins, user coins or network access tokens. The project would offer utility token to create resources for its intended expansion while the customer would purchase the right to utilize the product/ service created by the startup. An instance of a utility token is the Basic Attention Token (BAT). This token is created to be utilized as a compensation form inside the Brave browser in the perspective of publicity. Customers, publishers, and the promoters will use up the BAT token to intermingle with each other. Customers can utilize the BAT token to have entry to premium items and products, acquire high-quality photos and information as well as making contributions to suppliers of their preference.(21) Utility token provides holders of the token with the right to consume or access to the network or its product. However, it does not provide the right of ownership over a part of the company or project. (22)

\section{2) Equity Tokens}

Equity tokens offer the purchaser with component proprietorship of a venture. Equity tokens basically give the purchaser the right to a fraction of the venture. The ownership is in percentage to the number of tokens that have been purchased.(23) Equity tokens can be regarded as digital stocks in a business. In the United States, the recent ruling stated that these tokens fall under the purview of the Securities and Exchange Commission (SEC) and therefore subject to U.S. Securities Law.(24) any company that launches this type of token will have to ensure that they comply with the respective regulations. For investors, equity tokens are a attractive asset category because they ensure proprietorship which is patented in nature. An example of equity token is MCEX Security Token.(25)

\section{3) Asset Tokens}

The third type of token is known as asset token which is also described to as asset-backed tokens. These digital tokens are utilized to replace a tangible resource. If asset token is compared with utility token, asset token is able to provide a greater level of security because of the involvement of physical assets. For example, the Goldman project utilizes blockchain to digitize gold assets and to offer its asset-backed token. Asset tokens make it simpler to purchase real properties. However, they are becoming less prevalent since the value of a token is planned to be the equal as that of its tangible asset to which it is secured. (26)

\section{4) Reward Tokens}

Reward tokens are tokens that allow users holding inside an ecological unit.(27) By looking into the amount of reputation tokens a customer owns, it is able to ascertain the trustworthiness of the project. Customers will usually purchase these tokens established on involvement in a blockchain program. A good example of reward tokens is "rewards token" (RWTD). RWTD is an online marketplace for rewards which comprises merchants, millions of shoppers, discounts, coupons, products, and contests. Consumers can redeem their earned tokens anywhere in the ecosystem. Members receive access to the tickets and products before they go on sale to the public. RWRD tokens not only make the loyalty tokens fungible, but one can also trade them for cryptocurrency and also fiat currency. It allows users to earn their loyalty points through merchants worldwide. The token holders can also vote on proposals for promotion programs and special bonuses.(27)

There are different types and classifications of tokens. Each has its unique characteristics and offer different rights to its holder. Therefore, it is important for the potential investor to understand the unique nature and the various rights attached to different classes of the token. The rapid ICO surge in 2017 has resulted in the drafting and implementation of various regulations. The central bank stated tokens cannot be operated as exchange on the market and banks cannot extend services concerning ICOs.(28) Although tokens or cryptocurrencies are not recognized as currency in the market, however, in the United States, the Inland Revenue Service (IRS) has characterized cryptocurrencies as "property" rather than as a form of currencies for the purpose of tax consideration.(29) Cryptocurrency characterized as property is for the purpose of issuing initial guidance on matters relating to taxation, however if we evaluate this proposition from the legal point of view that ICO tokens are to be regarded as property, the question that we would like to ask is "how would this proposition assist potential investors when making an investment decision in ICO?" A potential investor would want to know what would be his/her greatest possible interest(s) or right(s) in an ICO token that he/she desires to invest. These interest(s) or right(s) are clearly explained in the concept of property. (30)

As such what is meant by 'property'? The conventional view of property is that it is a 'thing'. The shared perception is that property as a 'thing' in which the proprietor of such property has the absolute right of unrestricted usage, pleasure and discarding of it. (31) Property as a physical 'thing' cannot be applied in the context of cryptocurrency because cryptocurrency is intangible. (32) As new technologies rapidly develop and new creations emerge we need to establish an idea of property that will proficient to adjust the distinctive personality of these new creations. One should not confine the understanding of property base on the traditional property principles in that physical possession meant ownership in a property. John Maurice Clark, Social Control of Business $(1939,94)$ said:

"Generally individuals suppose of property as a quantifiable matter which someone holds. But the essential query is what is this idea we call possession? Possession comprises of a sizable and diverse package of privileges and rights." (33)

5) Hohfeld also articulated the subsequent words:

"Proprietorship [i]s a complicated set of lawful relationships in which people [a] are mutually dependent. Because proprietorship is interpersonal, no individual can possess comprehensive liberty to use up, retain, appreciate, or handover" their holdings, 
disputes, and impediments with rights are inevitable. The actual query in every argument is how judges make the value selections about which impediments with privileges should be banned or authorized." (34).

As illustrated by Hohfeld, proprietorship is a complicated set of lawful relationships and this explains how the owner of a property interacts with various parties that have some dealing with the property. If the interaction involves various parties, it implicates that the owner of the property holds more than one right in every property. For example, the proprietor of a car has a right to sell and a right to use the car. Owner may enjoy even more rights if the property in question is intangible, such as in copyright, the owner of the copyright has the right to access, the right to distribute and the right to reproduce.(35) Every property, whether tangible or intangible consists of various rights and the bundle of rights theory is the most appropriate property theory to describe and explain the various rights that exist in every property. (35)

The bundle of rights theory describes the possessions as an assortment or a bundle of rights that would permit in several aspects in which a property can be split up and appreciated by separate groups as to its connection between the groups that are plainly specified and well-handled. (36) In the bundle of rights, which is also known as the bundle of sticks theory, the property right or the stick in itself is property. The bundle of rights characterized property as a set of rights. Each One of the privileges in the bunch was impartial of each other.(36) Because of that, the bundle of rights theory was proficient to partition rights and decompose the freedom of usage to several groups. This involved that there is more than one person that could hold on or have been offered a right in the property. The theory stressed on parallel and numerous proprietorship as dissented to thing-based property idea that highlighted on single proprietorship in the property.(36)

Rights such as a right to determine how property must be utilized and design of all the appropriate characteristics of each user as well as the possibilities of such usage in every property are necessary. The importance of understanding 'property right' is that a right can be largely specified as a privilege or a title. (37) Rights can be imposed by intimidation or punishment or by the compensation. In numerous instances, there is no necessity for any kind of penalty as implementation on the foundation that many rights are usually recognized ethical principles. (38)

With a precise set of property rights, regulations and standards in place, it will avert and lessen the abuse and exploitation of property in the modern technological environment. Schlager and Ostrom have recognized five property rights that were highly applicable for the usage of common-pool assets involving admission, removal, supervision, omission, and disaffection.(39) Although we are not concern with common-pool assets in the current perspective, the five property rights can be used as a reference to identify the various rights in ICO tokens.

Admission: The right to join upon a specified physical area and experience non-subtractive advantages;

Removal: The right to delete assets.

Administration: The right to control inner usage designs and convert the resource by making upgrades. The right of administration is a collective-choice right allowing its owners to formulate operational-level removal rights regulating the usage of assets.

Omission: The right to decide who shall have admittance rights and removal rights and how those privileges may be reassigned;

Disaffection: The right to sell or rent. The right of disaffection is also a collective-choice right in which it allows its owners to transmit part or all of the collective-choice rights to a new person or company. (39).

One should note the differences between property rights and proprietary rights. They may have a similar meaning, but they represent two different categories of rights in the property. 'Property right' is described as a right that regulates the usage and control of an asset. When one is worried with the use and management of a thing or resource in question, it essentially implies to the right of that person in that thing or resource. However, on the other hand, 'proprietary right' is right that has proprietary character. Proprietary means ownership. The right 'in rem' in a property originally meant a right "in a thing". (40) This new idea gives the property its 'in rem' personality, that is, a right that is exclusive in nature which is enforceable against the world at large. Therefore, a property right includes every right that regulates the use and control of a resource i.e. the right to access and the right to use. However, not every property right is proprietary in nature which has the 'in rem' character.

There are only some property rights that are considered as a proprietary right and these rights are exclusively enjoyed by the owner of the resource. The right to manage the resource is an example of a proprietary right. The distinction between property rights and proprietary rights is crucial because it distinguishes one who is merely a holder of a particular right from the owner of a resource. For example, a person who only has a right to access is not the owner of a resource. The person is given a right to access the resource. That person has no authority to manage or to transfer such rights to another individual. However, the owner of a resource has the right to manage and deal with the resource according to the owner's wishes. The owner can grant temporary access or even withdraw the right of access to any person. This is a powerful position being an owner of a resource as opposed to merely as a holder of the property right in a resource.

Based on the above discussion, the aim of understanding the principles of property rights, a bundle of rights model and the distinction between property rights and proprietary rights is that these property principles would serve as a guide for the potential investors of ICO project. In considering whether to invest in a particular ICO project or not, it is very important for the potential investors to be able to identify all the relevant rights and benefits that are attached to the ICO tokens apart from considering the feasibility of the ICO project as a whole. There are three significant implications that can be derived from this proposition. First, the type of property rights and the number of rights attach to an ICO token would provide valuable information to the potential investors as to the true intrinsic value of the ICO tokens. The intrinsic value of ICO tokens 
also depends on the type of token that is in question. Secondly, a well-defined set of property rights for ICO tokens can serve as a reality check on the feasibility of the project. If an ICO token's bundle of right is not well-defined or does not provide a comprehensive explanation as to how the token should be used, specification of all the relevant attributes of each use and the contingencies of such use, then it reflects the flaws and inadequacy of the ICO project. The third implication is that if the property rights of ICO token are not properly defined, then it may be treated as an indicator of fraud because the provider of fraudulent ICO project would never be able to explain the various property rights in proper legal manner of an ICO project that he does not intend to carry out.

\section{B. Analysis of Various Property Rights of Actual ICO Tokens}

Flowing from the above discussion in relation to property rights and the bundle of rights theory, we should now examine how these theories are to be applied in actual ICO tokens offered in the market. For example, Reward Token (RWTD), there are various rights attached to the RWTD. The right to access to millions of shoppers, discounts, coupons, products, and contests. Holders of the RWTD with the designated tickets and products can have the right to enjoy special rewards before it goes on sale for the public. The right to manage allow holders of RWTD first to convert them for cryptocurrency and fiat currency, secondly, it allow the holders to have the right to participate in this program decide when and where to redeem their earned tokens anywhere in the ecosystem and thirdly right to vote on proposals for promotion and special bonuses which are actually collective-choice right authorising its holders to devise operational-level withdrawal rights if they think the proposed promotion programs or special bonuses are not acceptable. There are handfuls of property rights that have been given to the holders of RWTD. (27)

Doctor Smart Time Token (DSTT) includes technologies such as AI, telemedicine, and blockchain to the fields of healthcare and wellness management. Doctor Smart is based on a global blockchain ecosystem that will make affordable healthcare accessible to everyone. This ecosystem will also enable the integration of existing infrastructure, including hospitals and healthcare clinics while connecting care providers and clients worldwide.(41)

DSTT grants a partial digital right to participate in the Doctor Smart community, including some financial and non-financial privileges, right to access to better rates for patients and bigger functionality for doctors. Patients that own the DSTT will have the right to get additional discounts. On the other hand, doctors will be able to earn more as the platform will charge the doctors a smaller portion of their earnings. DSTT can also be used to pay for Doctor Smart Services. (41) The DSTT complies with ERC-20 standard, issued on the Ethereum blockchain and compatible with Quorum private blockchain, which is used in the Doctor Smart Ecosystem. The transfer of tokens between the two types of blockchains takes place without any restrictions or approvals. As a result of this transfer, the total number of tokens remains unchanged. (41)
DSTT grants the holder the right to access to Doctor Smart community, including some financial and non-financial privileges, right to access to better rates for patients and bigger functionality for doctors. It also grants the right of alienation which allows holders of DSTT to use it as a payment instrument, to pay for Doctor Smart services as well as the transfer of tokens.

Another ICO token to be discussed here is OmiseGO (OMG). OmiseGO is one of the most exciting projects to be built on top of etherum. Their goal is to become the world's biggest peer-to-peer cryptocurrency exchange platform that operates in Thailand, Japan, Singapore, and Indonesia. OmiseGo is created by Omise, which is separately operated as an extension platform. OmiseGO describes itself as: "The answer to a fundamental coordination problem amongst payment processors, gateways, and financial institutions". (42) OMG is similar to that of a decentralized bank, exchange, asset-backed blockchain gateway. OMG help the unbanked to get banking services through their open-wallet infrastructure and to facilitate unprecedented inter-changeability between many siloed legacy rails $(\mathrm{ACH}$, VISA, Paypal) and decentralized blockchains (such as Ethereum). (42) OMG allow holders to have the right to access to the world's biggest peer-to-peer cryptocurrency exchange platform, the right to alienation and the right to manage in which it allows holders to transfer and interact with other isolated payment systems.

\section{Operational and Governance Framework Design}

Another important area for the potential investor to read; study and scrutinize the ICO whitepapers will be the operational and governance framework design of an ICO project. A good ICO project should adopt requirements in line with the good governance and framework to help to ensure the quality of ICOs offer in the market. (43) Although this area is highly technical and potential investors may not have the opportunity to view the source code of the ICO project, nevertheless, potential investors should demand a high quality of disclosure from the provider of ICOs on the operational and governance aspect. Providers of ICOs should invite and encourage dialogue and challenge with potential investors so that their ICO whitepapers can be vetted and tested accordingly. However, many projects do not encourage dialogue with no forum for open discussion. Providers of ICOs may even intentionally hide information and the source code from potential investors. Potential investors should try to search for the source code of an ICO project from the Github page in which it is a repository where teams or individuals will upload code to public or private accounts. Github is an open source community placing creative people together on an open platform, to connect developers around the world to ask questions, swap stories, and share ideas on public or private projects. When the teams of the ICO projects choose to make the code open source in Github, it means that they welcome the community to contribute to the project. When a team is very active in Github, this actually represents a very good sign because it shows that programmers behind the ICO project are spending time working on the project and to

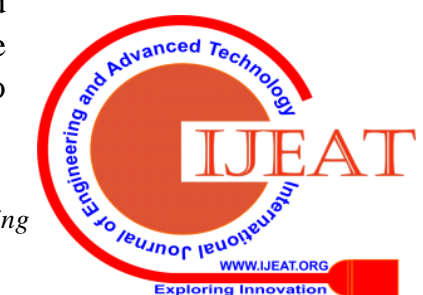


interact with developers around the world to improve on it. (44) This probably suggests the ICO project is a genuine project because if it is a scam ICO project, the providers would not want to spend time interacting with other developers in Github which will eventually expose their fraudulent project in Github forum.

Furthermore, if the provider of ICO and his team have published their token smart contract codes, developers who are well versed in this area can verify whether a smart contract matches the claims made in the white paper. The following are some of the questions that potential investor should ask when they read whitepapers of ICO:

- Does the token provide access to a product, give interaction rights to it, or grant the right of ownership to a value?

- Does the token offer the opportunity to govern - for example, the right to vote or to have an impact on decision-making and/or the development of a new product or service?

- Is there the possibility to add value to the token in the network or relevant market, or to receive rewards for active work?

- Does the token serve as a security deposit, a principal payment unit, or the principal accounting unit to buy and sell things, or is it needed to join a network or any related entity?

- Does a designated blockchain autonomously distribute profits and other benefits to token-holders?

- If the token is claimed as a utility, does the project have any business strategies to prevent project cash-flow gaps due to the volatility of tokens and crypto?

There are many more questions to be asked by potential investors. However, in order to ask the right questions for an ICO project, one must first identify the types of token offered in ICO and the type of property rights that are attached. As we can see from the above list of questions, in fact, most questions are concerned with 'what can one do with the token?'. More specifically, as a holder or owner of a token, what kind of right(s) so he/she has? For example, if the ICO offers reward tokens, then the reward token should give holder property rights such as the right to access a resource system, the right to receive or to earn a reward and also the right to manage the reward token. More importantly, the smart contract code should be written in such a way that corresponds with these rights and its execution. If the smart contract does not provide proper coding and algorithms to reflects and allow execution of those rights, then there is something wrong in this ICO project which the potential investor must take note of. (44) Furthermore, smart contracts and token issuance platforms should at least comply with the ERC-20 token standard and to be able to appreciate or identify the various basic functions in the source code if the code is available.

There are many different types of smart contract platforms; Ethereum blockchain-based ICOs accompanied by smart contracts or dApp are the most popular platform. (45) The tokens issuance program is expected to be written in conformity with the ERC Standard. ERC stands for Ethereum Request for Comment and it is an adaption of the Internet Engineering Task Force (IETF) Request for Comment (RC) standard. Numbers are appended to ERC e.g. ERC-20 in order to distinguish them from each other.

The ERC standards vary and are made for different aspects of the Ethereum Blockchain architecture. (46) For the purpose of looking into the operational and governance framework design, we are only concerned with the final Token Standards ERC-20 of which the ICO tokens are expected to be based on. And due to this, it is important for investors to verify if the tokens of the ICO are compliant to the approved token standards.

Among the token standards, the first and the most used is the ERC-20 standard authored by Fabian Vogelsteller and VitalikButerin. (46) This standard describes several functions that a smart contract must implement in order to be classified as a token by these applications (such as wallets, exchanges, and other contracts expecting to interact with tokens). (24) If potential investors are allowed to check on the source code of the token, they would be able to see whether the implementation of the governance framework conforms to the ERC standard in question.

With reference to the ERC-20 token standard, the ICO Token smart contract should implement three optional rules, six mandatory rules, and two events.

\section{Optional rules are:}

$$
\begin{aligned}
& \text { - } \text { name() } \\
& \text { - } \quad \text { decimbol() } \\
& \text { - decimals() }
\end{aligned}
$$

The name function is forgetting the name of the token, symbol for getting the token symbolic representation of the token, decimal for getting the number of decimals of the token.

\section{Six Mandatory Rules are:}

\section{Total Supply ()}

The total supply is for getting the total supply of the token in the ecosystem. (47)

// Get the total token supply

function total supply () constant returns (uint256 total supply)

2. balanceOf (address_owner)

balance is for getting the account balance of another address associated with the token

// Get the account balance of another account with address _owner

function balance (address _owner) constant returns (uint256 balance)

3. Transfer (address_to, uint 256 _value)

The transfer is for transferring amounts to an address. function transfer (address _to, uint 256 _value) returns (bool success)

// Send the _value amount of tokens from address _from to address _to

4. TransferFrom(address_from, address _to, uint 256 _value)

transfer from is for transferring from one address to another function transferFrom(address _from, address_to, uint256 _value) returns (bool success)

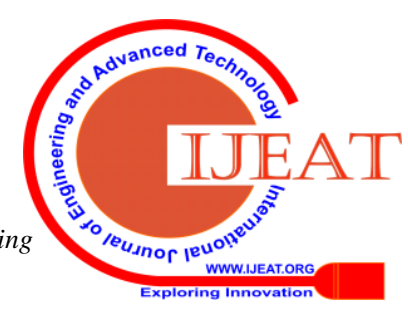


// Allow _spender to withdraw from your account, multiple times, up to the _value amount.

// If this function is called again it overwrites the current allowance with_value.

5. Approve (address_spender, uint 256 _value)

approve for granting approval to an address to withdraw multiple times up to the limit stated from an account function approve (address _spender, uint 256 _value) returns (bool success)

// Returns the amount which _spender is still allowed to withdraw from_owner

Allowance (address_owner, address_spender)

allowance with gives the balance of the amount the approved account can still withdraw.

function allowance (address _owner, address _spender) constant returns (uint256 remaining)

// Triggered when tokens are transferred.

\section{Two Events}

In addition to the functions, there are also two events that should be implemented and triggered by the token contract.

1. Transfer (address, address, uint256)

event Transfer (address indexed_from, address indexed _to, uint256_value)

// Triggered whenever approve (address _spender, uint256 _value) is called.

Approval (address, address, uint256)

2. Event Approval (address indexed_owner, address indexed_spender, uint 256 _value)

Tokens contracts are expected to trigger the Transfer event when they create new tokens and upon any successful call to approve function the Approval event must also be triggered. (47)

Although the six mandatory rules and the two events of ERC-20 token standard are quite simple, straight forward and require the various functions to be implemented in a smart contract and token issuance platform of ICO projects, when these six mandatory rules and the two events read together with the property rights as elaborated above in this research paper, they are able to provide a comprehensive guide for potential investor in scrutinizing the operational and governance framework design of an ICO project. This is how it works: Assuming one is looking into an ICO project that issues reward tokens, potential investors should first ascertain the total numbers of token to be issued by the ICO project. The points to be considered are whether the issuance of the token is exhaustive or non- exhaustive in nature. If it is exhaustive, then what will be the total numbers of token to be issued and the condition that needs to be fulfilled? If it non-exhaustive in nature, what will be the schedule for the issuance of token and the conditions that need to be fulfilled? Will the conditions be changed from time to time? Will the conditions also affect the rights that have been granted in the first place?

A potential investor must then identify the various rights that are to be granted from the token. If the right to access is to be granted to the reward system that consists of various companies, shops around the world, the potential investor must enquire about the exact rights and privileges that come together with such access. What are the conditions that need to be fulfilled and how the token to be transferred when the

reward is earned? Potential investors must pay close attention to the 2 mandatory rules for transfer functions as well as the event transfer in the smart contract. The whitepaper of the ICO project or its source code should clearly explain, illustrate and reflect on how the transfer of token takes place through various transactions in relation to the reward system.

If the right to manage is granted to the token holder, the questions to be asked are what are the rights? Does it allow the holders to participate in this system and to decide when and where to redeem their earned tokens in the system and how do the transfer of token take place? Apart from what has been stated above in relation to the transfer rules, another aspect potential investor may pay attention is does the code provide the allowance rule in which the allowance function is to check when a user executes a transfer function, whether he/she has the minimum required of the token. If the user does not have the minimum number of tokens, the transaction should be canceled. (47) Furthermore, will the transfer of tokens require approval from the system? The approve function is to check the transaction against the total supply of tokens. This function is to make sure that there is no missing or extra token. This function further ensures that there is no counterfeiting of token take place. These are the very crucial operational and governance functions that ought to be presented in every framework of an ICO project. If these functions are not in place, it only reflects that either the provider or the developer of the ICO project never thought about it or the developer did not write the code consistent with what has been stated in the whitepaper. (48) A code reflects on the attitude of a developer and also the provider of an ICO. If they are very serious and committed to the ICO project, they will ensure the quality and consistency of the codes that correspond to the ICO project. Therefore, if the potential investor does not see clearly the transfer or approve functions in the source code, then they should raise their concern to the provider of the ICO project and ask for further explanation and clarification.

The next question is whether the right to manage an ICO token also includes the right to withdraw from the system? If yes, what are the conditions attached to the withdrawal? In the situation when a token holder no longer wants to participate further in this reward system, does he/she possess the right to withdraw and convert his/her remaining token for cryptocurrency or fiat currency? The right to withdraw normally corresponds to the right to vote. If the token holder has given the right to vote on proposals for promotion and special bonuses, does he/she also have the right to withdraw if they think the proposed promotion programs or special bonuses are not acceptable? Recently at Devcon4, the annual developer conference for Etherum, the developer Fabian Vogelsteller, who formalized the ERC-20 token standard, proposed a way to allow investors to take back their funding from an initial coin offering. This new funding is called a "reversible ICO" or RICO. (49) This new funding allows investors to have the choice to withdraw their contribution at any time of the lifespan of the project. Fabian Vogelsteller stated that:

"You are able to withdraw the funds you committed at any point

Published By: 
of time and you do this by simply sending back your token" (49)

Therefore, when the potential investor is considering an ICO project, he/she must look out for the right of withdrawal in the event that he/she is no longer wants to participate in this reward system or an ICO project. The new funding proposed by Fabian Vogelsteller as explained above would be the most attractive as the holder of the token can just sending back the token to the provider of the project. But not all projects would adopt this new funding structure. Therefore, it is important for a potential investor to ask first is there a right to withdraw and what would be the necessary procedure that allows converting the remaining token for cryptocurrency or fiat currency in the event that the holder of the token decides to withdraw from the system.

As stated in the earlier part of this research paper, we draw a distinction between property rights and proprietary rights. Every token would possess the unique bundle of property rights but not every property right in the bundle is proprietary in nature. ICO token such as equity token and asset token would grant holder proprietary rights on part ownership of a project, signify or substitute a physical asset. When physical asset involves, it is important for a potential investor to understand how the token represents and correlated with an external, real-world asset's value. (50) Furthermore, potential investors should ask what would be the procedures for liquidation of a token. Is there any additional condition such as holder require to hold the token for more than a year or so forth? (51)

Base on the above analysis, an interesting observation that can be made is that from the outset, one would think that the property rights, operational and governance framework design are very different and they are not related to one another. However, when one examines the six mandatory rules together with the various property rights, each of the mandatory functions facilitates the access, management, and transfer of those rights. In fact, the functions and the rights work hand in hand and are inter-related with one another. The functions to be implemented and triggered by the token contract should correspond with the type of token involved and the property rights that are granted by the token provider. All relevant attributes of use as well as the contingencies of use of every token and its rights should be clearly explained in the ICO whitepapers. If the smart contract code does not provide proper and corresponding coding and algorithms to reflects and allow execution of those functions and rights, then there is a serious issue in an ICO project.

\section{CONCLUSION} blinded with the promise of tremendous returns in ICO whitepapers. They must understand that the development of ICO project is still in a very early stage and that it is highly uncertain whether the promised plans can be executed and realized. The only thing that the investors can rely on is the details that are disclosed in the ICO whitepapers. Potential investors should rightly insist on high quality, truthful and proper disclosures of all relevant information from the provider of ICOs on the legal, operational and governance
All potential investors should not let themselves to be

aspects. A potential investor should first identify the bundle of property rights in an ICO token. Potential investors should further look into the mandatory rules as stated in ERC-20 token standard together with the property rights that are incidental to the issuance and usage of the token in question. The coding and algorithms will serve as a checklist as to whether the provider provides a property framework or system that delivers what they promised and reflect an adequate level of operational governance of the project. Although looking at the coding and algorithms are highly technical, but high quality and consistent coding serve as good indicators of an ICO project. If potential investor carefully studies and scrutinizes ICO papers, they will not be susceptible to over promise, misrepresentation, fraud, and manipulation. When potential investors are getting smarter in evaluating ICO whitepapers, this is a sign of a mature market and investors are looking for classic and substantive criteria such as the team, the token value, the operational governance as well as the source code in making their investment decision. All these contribute towards the building of a better mechanism or platform for funding of projects with great potential in an unprecedented way.

\section{REFERENCES}

[1] European Securities and Markets Authority. ESMA highlights ICO risks for investors and firms. WwwEsmaEuropaEu. 2017;33(November):1-3.

[2] Juliano A. dYdX: A Standard for Decentralized Derivatives 2017;1-18

[3] Mehraban LS, Szczepanik VA, Tenreiro JG. SEC Complaint: REcoin Group Foundation, LLC, DRC World Inc. a/k/a Diamond Reserve Club, and Maksim Zaslavskiy. :1-24.

[4] Afm, T., Offerings, I. C., Ico, A., \& At O. Initial Coin Offerings ( ICO's ): serious risks. 2018;1-6.

[5] Lee J. Patch transporter: Incentivized, decentralized software patch system for WSN and IoT environments. Vol. 18, Sensors (Switzerland). 2018.

[6] Juliano A. dYdX: A Standard for Decentralized Derivatives.

[7] Investopedia. Distributed Ledgers Definition. 2018.

[8] Thake M. What's the difference between blockchain and DLT? Medium. 2018.

[9] BIS. V . Cryptocurrencies : looking beyond the hype. Annu Econ Rep 2018;91-114.

[10] Tipton H, Krause M, Ikbal J. An Introduction to Cryptography. Information Security Management Handbook, Sixth Edition. 2010. $1121-1140 \mathrm{p}$.

[11] Rouse M. What is micorcontroller_- Definition from WhatIs.

[12] Miana F. Mixing Symbolic and Ternary Simulation Techniques for the Verification of Processor-Based Systems. 1857;31-9.

[13] Citowise. The Basics: Coin vs. Token. What is the Difference? Medium.Com. 2018;

[14] Krawisz D. Bitcoin as a Store of Value, Unit of Account, and Medium of Exchange | Satoshi Nakamoto Institute. Nakamoto Institute. 2015.

[15] Pietrewicz L. Emerging Trends in Entrepreneurial Finance: The Rise of ICOs. Stud i Mater Wydz Zarządzania UW 2018;1/2018(27):65-78.

[16] Bramanathan R. The perfect token sale structure - The Coinbase Blog Blog.Coinbase.Com. 2017. p. 4.

[17] Zhao W. Tea Tokenizers Arrested in China for Alleged \$47 Million Crypto Fraud - CoinDesk. 2018.

[18] CoinTelegraph. What Is A White Paper And How To Write It? Coin Telegraph. 2018;

[19] Zetzsche DA, Buckley RP, Arner DW, Föhr L. The ICO Gold Rush: It's a Scam, It's a Bubble, It's a Super Challenge for Regulators. SSRN. 2017.

[20] Wilmoth J. 3 Types of ICO Tokens - Strategic Coin Strategiccoin.Com. 2018.

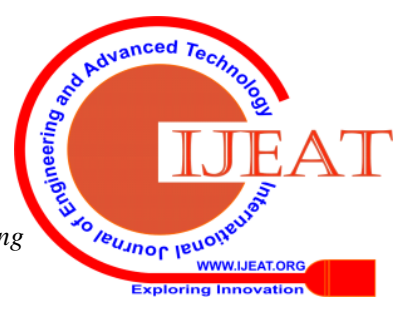


[21] Basic Attention Token.

[22] Wei WX. Rewards Token Ico Brave Coin Ico. 2019.

[23] Reder M. What is Equity Token Offering (ETO) and why is it safer than every ICO ever created_. 2018.

[24] Uiuc) (, Saxena M. KEVM: A Complete Semantics of the Ethereum Virtual Machine. RV Inc; 2017.

[25] Exchange MC. SECURE TOKEN.

[26] James. A guide to gold-backed cryptocurrency. Goldscape.net. 2018.

[27] Rewards Token (RWRD) ICO Review - ICO Token News.

[28] Investopedia. Initial Coin Offering (ICO). Investopedia LLC. 2017. p. $1-8$.

[29] Lee IKF\& J. IRS Makes Cryptocurrency a Compliance Priority Lexology.

[30] Hall C. Ownership. Vol. 32, Air Medical Journal. 2013. p. 192.

[31] Wenar L. The concept of property and the takings clause. Columbia Law Rev. 1997;97(6):1923.

[32] EYGM. IFRS (\#) Accounting for crypto-assets. 2018;1-22.

[33] Klein DB, Robinson J. Property: A bundle of rights? Prologue to the property symposium. Econ J Watch. 2011;8(3):193-204.

[34] HOHFELD W. "Some Fundamental Legal Conceptions as Applied in Judicial Reasoning." Canon Am Leg Thought. 1913;23(1):45-82.

[35] N M A, Aswath L. Understanding Copyright Laws: Infringement, Protection and Exceptions. Int J Res Libr Sci. 2016;2(May):2455-104X

[36] Johnson DR. Reflections on the Bundle of Rights. Vt L Rev. 2007:32:247.

[37] 3Merges RP. What Kind of Rights Are Intellectual Property Rights? $2017 ; 1$.

[38] Dworkin R. Natural Law Revisited. 1982;

[39] Schlager E, Ostrom E. Property-Rights Regimes and Natural Resources: A Conceptual Analysis Property-Rights Regimes and Natural Resources : A Conceptual Analysis Edella Schlager and Elinor Ostrom property rights and the rules used to create. Land Econ. 2010;68(3):249-62.

[40] Singer JW. Property as the Law of Democracy. Ssrn. 2014;

[41] Doctor Smart (DSTT) ICO Review - ICO Token News.

[42] What is OmiseGO \& The Plasma Protocol_ - Blockgeeks.

[43] Waters T, Maywald IM. ICOs: A call for governance - Ashurst. 2017.

[44] Fiedur B. How to spot good ICO, how to spot scam ICO - Hacker Noon. 2018;

[45] Tsng M. Token Issuance (ICO) Best Practices and Resources. 2017.

[46] ERC_Ethereum Improvement Proposals.

[47] Blockgeeks. ERC20 Tokens: The Origin Story (Blockgeeks Guide).

[48] Mulders M. 10 keys for evaluating Initial Coin Offering (ICO) investments. CryptoPotato. 2017.

[49] Krystle M. Ether Developer Proposes a _Reversible ICO_ to Allow Crypto Tokens Investors to Withdraw Commitment. 2018.

[50] Curran B. What is an STO- A Complete Guide to Security Token Offerings. Https://Blockonomi.Com/What-Is-an-Sto/. 2015.

[51] Siegel D. Equity Token Finance - Hacker Noon. 2017.

\section{AUTHORS PROFILE}

I am Wong Wai Wai, affiliated with University of Malaya, Faculty of La. For further details contact drwongwaiwai@gmail.com

My name is Muhammed Siraj, and my affiliation is Universiti Putra Malaysia.my area of interest is governance. 